\title{
Síndrome de Hallermann-Streiff y embarazo: manejo de la vía aérea difícil en embarazadas
}

\author{
Andrés Rojas G. MSc 1,3,4, Marco Balkenhol N. 1,3,4, Omar Herrera C. 1,3, Marcela \\ Opazo V.2,3, Marcela Hernández P.1,3,4, Marcos Rivera C. 1,3,4. \\ ${ }^{1}$ Servicio de Anestesiología. ${ }^{2}$ Servicio de Medicina Interna. ${ }^{3}$ Hospital de Puerto Montt. ${ }^{4}$ Universidad San Sebastián. \\ Puerto Montt, Chile.
}

\section{RESUMEN}

El síndrome de Hallermann-Streiff es una rara entidad asociada a hipoplasia del tercio inferior de la cara, determinando así una vía aérea de difícil manejo. Se presenta el caso de una mujer de 21 años con ese síndrome, acondroplasia, escoliosis severa e infección respiratoria los días previos a la interrupción exitosa de su embarazo mediante cesárea. El manejo requirió una cuidadosa evaluación preoperatoria y disponibilidad inmediata de dispositivos alternativos para el manejo de la vía aérea en caso necesario. La gravidez, así como ciertas comorbilidades asociadas, aumentan la posibilidad de una intubación fallida con morbimortalidad secundaria importante, dado ello, es necesaria la preparación e implementación de algoritmos atingentes para el manejo de la vía aérea en casos como el presentado.

\section{PALABRAS CLAVE: Síndrome de Hallermann-Streiff, embarazo de alto riesgo, manejo de vía aérea, anestesia}

\section{SUMMARY}

The Hallermann-Streiff syndrome is a rare entity associated to hypoplasia of the lower third of the face, determining a difficult airway management. We report the case of a 21 years female with this syndrome, achondroplasia, severe scoliosis and respiratory infection at the days prior to the interruption of her pregnancy by cesarean section. Her management required a carefully preoperative evaluation and availability of alternative devices to secure her airway. Pregnancy and certain comorbidities increase the chance of a failed intubation with severe secondary morbidity and mortality, given this, the correct preparation and implementation of difficult airway algorithms in pregnancy if it's necessary.

KEY WORDS: Hallermann-Streiff syndrome, high-risk pregnancy, airway management, anesthesia

\section{INTRODUCCIÓN}

El síndrome de Hallermann-Streiff, o discefalia de Francois, es una rara entidad asociada a hipoplasia del tercio inferior de la cara (facie "tipo pájaro"), dentición anormal, hipotricosis, cataratas congénitas y acondroplasia (1). Estos hallazgos, sumados al estado de gravidez per sé, determinan condiciones de riesgo tanto para la madre como feto, al dificultar el control de la vía aérea sobre todo en situaciones de emergencia (2). Dado lo anterior, y considerando el progresivo aumento de embarazadas con comorbilidades asociadas, es necesario conocer algunos aspectos potencialmente modificables que pudiesen disminuir el riesgo de un desenlace fatal para la unidad materno-fetal, secundaria a una intubación y/o ventilación fallidas. 


\section{Caso clínico}

Paciente de 21 años, $39 \mathrm{~kg}$ de peso, $101 \mathrm{~cm}$ de talla. Portadora de síndrome de HallermannStreiff diagnosticado a los 5 años de vida. Escoliosis severa, hidrocefalia, anemia leve y diabetes gestacional en control dietético. Presenta predictores de vía aérea difícil como micrognatia, distancia tiro-mentoniana de $5 \mathrm{~cm}$, apertura bucal de $2 \mathrm{~cm}$ y Mallampati 2. Con fecha de interrupción para las 38 semanas debido a desproporción céfalo-pélvica, es hospitalizada a las 35+2 semanas de embarazo por bronco-obstrucción marcada de probable etiología viral (se descartó asma mediante espirometría), con buena respuesta a oxigenoterapia, broncodilatadores, prednisona (40 mg/día) y kinesioterapia. Durante la hospitalización, y cursando $36+4$ semanas, inicia trabajo de parto, motivo por el cual se realiza cesárea no programada. Dada la complejidad técnica de un abordaje anestésico neuroaxial determinada por su severa escoliosis y cono medular de implantación baja (Figura 1), se decide administrar anestesia general. Preoxigenación con O2 100\% por 3 minutos para posterior inducción en secuencia rápida con propofol $80 \mathrm{mg}$, lidocaína 40 $\mathrm{mg}$ y succinilcolina $40 \mathrm{mg}$ EV. Al momento de la intubación se realiza maniobra de Sellick constatándose laringoscopia grado 3 de Cormack-Lehane, introduciendo tubo orotraqueal 5.5 mediante apoyo de bougie, sin incidentes y en un intento. Como dispositivo alternativo en caso de fallo intubación se contaba con máscara laríngea clásica, no contando con videolaringoscopio ni entrenamiento para intubación vigil mediante fibrobroncoscopia. Tiempo total de intervención fue de 30 minutos, manteniéndose anestesia mediante $\mathrm{O} 2$ 50\% más N2O 50\% y sevoflurano a concentración alveolar mínima de 0,9 , presiones de vía aérea en rangos normales, capnografía con patrón obstructivo y oximetría de pulso $\geq 95 \%$. Hemodinamia siempre estable. Tras 20 minutos se realiza extracción de recién nacido de sexo femenino, sin malformaciones visibles, peso $2315 \mathrm{~g}$, con adecuada edad gestacional (percentil 13 y 36 semanas por examen físico) y Apgar 8 puntos al minuto y 9 puntos a los 5 minutos de vida. Se administró fentanyl $50 \mu \mathrm{g}$ más morfina 2 mg EV posterior a ligadura de cordón. Se realiza esterilización quirúrgica mediante salpingoligadura de Parkland, dejándose analgesia endovenosa continua con metamizol y morfina. En el postoperatorio se mantiene sin complicaciones respiratorias, con buen control hemodinámico y analgésico. Ambas son dadas de alta al tercer día sin complicaciones.

\section{DISCUSIÓN}

Este síndrome fue descrito por Aubry en 1893 y luego en 1948 por Hallermann y Francois

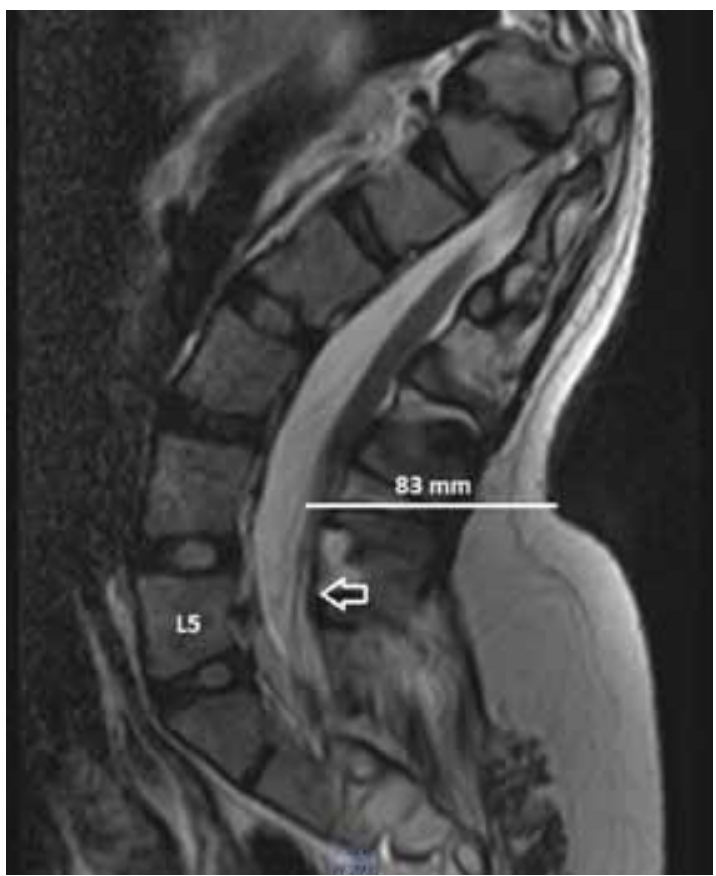

Figura 1. Vista sagital de resonancia magnética en modo TW2 de columna lumbosacra mostrando escoliosis severa, cono medular de implantación baja (flecha) y distancia mayor a $8 \mathrm{~cm}$ desde piel hacia espacio epidural.

siendo la mayoría de los casos esporádicos $(1 / 27.472$ nacidos vivos) $(3,4)$. Su etiología no es clara, proponiéndose una falla en el desarrollo del segundo arco branquial en relación a infecciones virales maternas, gestación tardía y exposición a toxinas (¿tetraciclina?) (4). Pueden presentar disnea, apnea obstructiva del sueño, cardiopatías, infecciones pulmonares recurrentes y paro respiratorio pos-anestesia. Dada su inteligencia normal, tienen una mayor chance de embarazarse con respecto a otras pacientes con dismorfia y retardo mental asociado, reportándose en los últimos 30 años casos aislados pero exitosos de parto normal así como de cesárea (1).

Anestesia obstétrica y mortalidad materna: No existen reportes de mortalidad materna en estas pacientes, estimándose en líneas generales en $0,12 / 100.000$ recién nacidos vivos para anestesia en cesárea, siendo la mayoría de los casos en un contexto de urgencia similar al nuestro (5). La Tabla I resume los múltiples factores que explican los riesgos maternos asociados a la pérdida del control de la vía aérea. 


\section{Tabla I \\ CAMBIOS FISIOLÓGICOS SECUNDARIOS AL EMBARAZO ASOCIADOS A MAYOR RIESGO DE VÍA AÉREA DIFÍCIL}

\begin{tabular}{|c|c|c|c|}
\hline Riesgo & Cambio fisiológico & Consecuencia & Manejo \\
\hline \multirow{3}{*}{ Vía aérea difícil } & $\begin{array}{l}\text { Reducción } \mathrm{CRF}^{\mathrm{a}} \text { y mayor } \\
\text { consumo } \mathrm{O} 2\end{array}$ & $\begin{array}{l}\text { Desaturación rápida en } \\
\text { apnea }\end{array}$ & Preoxigenación adecuada \\
\hline & $\begin{array}{l}\text { Edema tisular e hiperemia } \\
\text { mucosa }\end{array}$ & $\begin{array}{l}\text { Obstrucción por intentos } \\
\text { fallidos }\end{array}$ & Dispositivos alternativos \\
\hline & $\begin{array}{l}\text { Aumento volumen } \\
\text { mamario }\end{array}$ & $\begin{array}{l}\text { Dificultad inserción } \\
\text { laringoscopio }\end{array}$ & $\begin{array}{l}\text { ¿Posición en rampa? (en } \\
\text { obesas) }\end{array}$ \\
\hline \multirow{2}{*}{ Aspiración } & $\begin{array}{l}\text { Atonía esfínter esofágico } \\
\text { inferior }\end{array}$ & $\begin{array}{l}\text { Regurgitación contenido } \\
\text { gástrico }\end{array}$ & $\begin{array}{l}\text { Aspiración contenido } \\
\text { gástrico por sonda }\end{array}$ \\
\hline & $\begin{array}{l}\text { Aumento niveles } \\
\text { progesterona }\end{array}$ & $\begin{array}{l}\text { Retardo vaciamiento } \\
\text { gástrico }\end{array}$ & $\begin{array}{l}\text { Procinéticos y ayuno } \\
\text { adecuado }\end{array}$ \\
\hline \multirow{2}{*}{$\begin{array}{l}\text { Despertar intraoperatorio } \\
\text { (o awareness) }\end{array}$} & $\begin{array}{l}\text { Rápida redistribución } \\
\text { agente endovenosos }\end{array}$ & $\begin{array}{l}\text { Riesgo hipotensión, } \\
\text { depresión neonatal y atonía } \\
\text { uterina }\end{array}$ & $\begin{array}{l}\text { Reducción dosis fármacos, } \\
\text { monitoreo sedación } \\
\left(\mathrm{BIS}^{\mathrm{b}}, \mathrm{EEG}^{\mathrm{C}}\right)\end{array}$ \\
\hline & $\begin{array}{l}\text { Equilibrio lento de agentes } \\
\text { volátiles }\end{array}$ & $\begin{array}{l}\text { Riesgo hipotensión, } \\
\text { depresión neonatal y atonía } \\
\text { uterina }\end{array}$ & $\begin{array}{l}\text { Reducción dosis fármacos, } \\
\text { monitoreo sedación } \\
\left(\mathrm{BIS}^{\mathrm{b}}, \mathrm{EEG}^{\mathrm{C}}\right)\end{array}$ \\
\hline
\end{tabular}

a: Capacidad Residual Funcional. b: BISpectral Index. c: Electroencefalograma

Embarazo y aspiración: El riesgo de aspiración en cesárea es tan bajo como $0,0625 \%$ pero potencialmente mortal, dado ello, se recomienda la administración 30 minutos previo a la cesárea de algún procinético y antiácido, inclusive durante la intubación misma si es urgente (3). Un tercio de las aspiraciones ocurren durante la extubación, sugiriéndose previamente la aspiración gástrica mediante sondaje (3). Con respecto a la presión cricoidea (Sellick) durante la intubación en casos de alto riesgo aspirativo, la evidencia es dispar en cuanto a su real efectividad (6), impresionando más bien que la masificación de la intubación en secuencia rápida en estos casos explicaría la reducción en la morbimortalidad materna en urgencia (7).

Preoxigenación: Durante el embarazo, el periodo en apnea sin desaturación es de 1-2,5 minutos (8), dado ello la preoxigenación es esencial, siendo óptima cuando el $\mathrm{O} 2$ espirado es $\geq 90 \%$ administrando $\mathrm{O} 2$ al $100 \%$ a volumen corriente $7 \mathrm{ml} / \mathrm{kg}$ por 3 minutos u 8 respiraciones a capacidad vital por 1 minuto. La posición en rampa, como maniobra de preoxigenación, no ha demostrado beneficios en este tipo de pacientes pero pudiese mejorar la laringoscopia sobretodo en obesas (5).

Inducción y mantención de la anestesia para cesárea: Propofol $(2-2,5 \mathrm{mg} / \mathrm{kg})$ genera inconciencia en \pm 30 segundos, disminuye la reactividad de la vía aérea, permite rápido despertar y reduce incidencia de náuseas y vómitos, no comprometiendo el flujo sanguíneo uterino e inclusive pudiendo disminuir el riesgo de atonía uterina (8). Ketamina (1-1,5 $\mathrm{mg} / \mathrm{kg})$ suma propiedades simpaticomiméticas en caso de hipotensión o hiperreactividad bronquial. Etomidato $(0,2 \mathrm{mg} / \mathrm{kg})$ se reserva en el contexto de cardiopatía o inestabilidad hemodinámica (9).

La adición de benzodiacepinas disminuye la probabilidad de despertar intraoperatorio, mientras que los opioides de acción corta como fentanyl o remifentanilo, a pesar de su potencial asociación a depresión neonatal, disminuyen el riesgo de awareness, estabilizan la respuesta hemodinámica a la intubación y reducen la liberación de catecolaminas asociadas a dolor que podrían afectar la perfusión uterina (10). Para la mantención de la anestesia se recomienda un halogenado a una CAM 0,7-1,0 pues valores mayores se asocian a atonía uterina con o sin administración de óxido nitroso (9).

Relajantes musculares: En caso de no intentar una intubación vigil, la succinilcolina $(1-1,5 \mathrm{mg} / \mathrm{kg}$ EV) permite relajo muscular en \pm 30 segundos debido a su rápido volumen de distribución en embarazadas, dado ello, no se recomienda precurarizar por riesgo de bloqueo parcial y enlentecimiento en su acción (6). En caso de contraindicación, rocuronio 
(1,2 mg/kg EV) ha sido propuesto también para intubación en secuencia rápida, con la ventaja adicional de contar con antídoto (sugammadex 2-16 mg/kg) lo cual permite su reversión en 2 minutos en caso necesario (7).

Vía aérea difícil en embarazada: La incidencia de intubación fallida en pacientes obstétricas se estima en 1:300, 10 veces más que en la población general (11), lo cual se explica en parte dado que un 1/3 presentarán uno o más predictores de intubación difícil. Al respecto, destacar que ningún predictor per sé es suficiente en estas pacientes para catalogarlas como vía aérea difícil, inclusive avances recientes como la medición ecográfica de la sombra acústica subglótica retrotraqueal, no han mostrado correlación clínica, dado lo anterior, su detección deberá basarse en una combinación de predictores clínicos (11).

Debido a estas particularidades, diversos grupos han desarrollado algoritmos de enfrentamiento de vía aérea difícil en la embarazada $(11,12)$ (Figura 2), considerando en sus fundamentos el estado de la unidad materno-fetal y la disponibilidad de dispositivos alternativos para el manejo de la vía aérea como lo recomienda la American Society of Anesthesiologists (13).

\section{Monitorización / Ayuda / Carro via aérea dificil / Informar a obstetra / Definir urgencia interrupción embarazo}

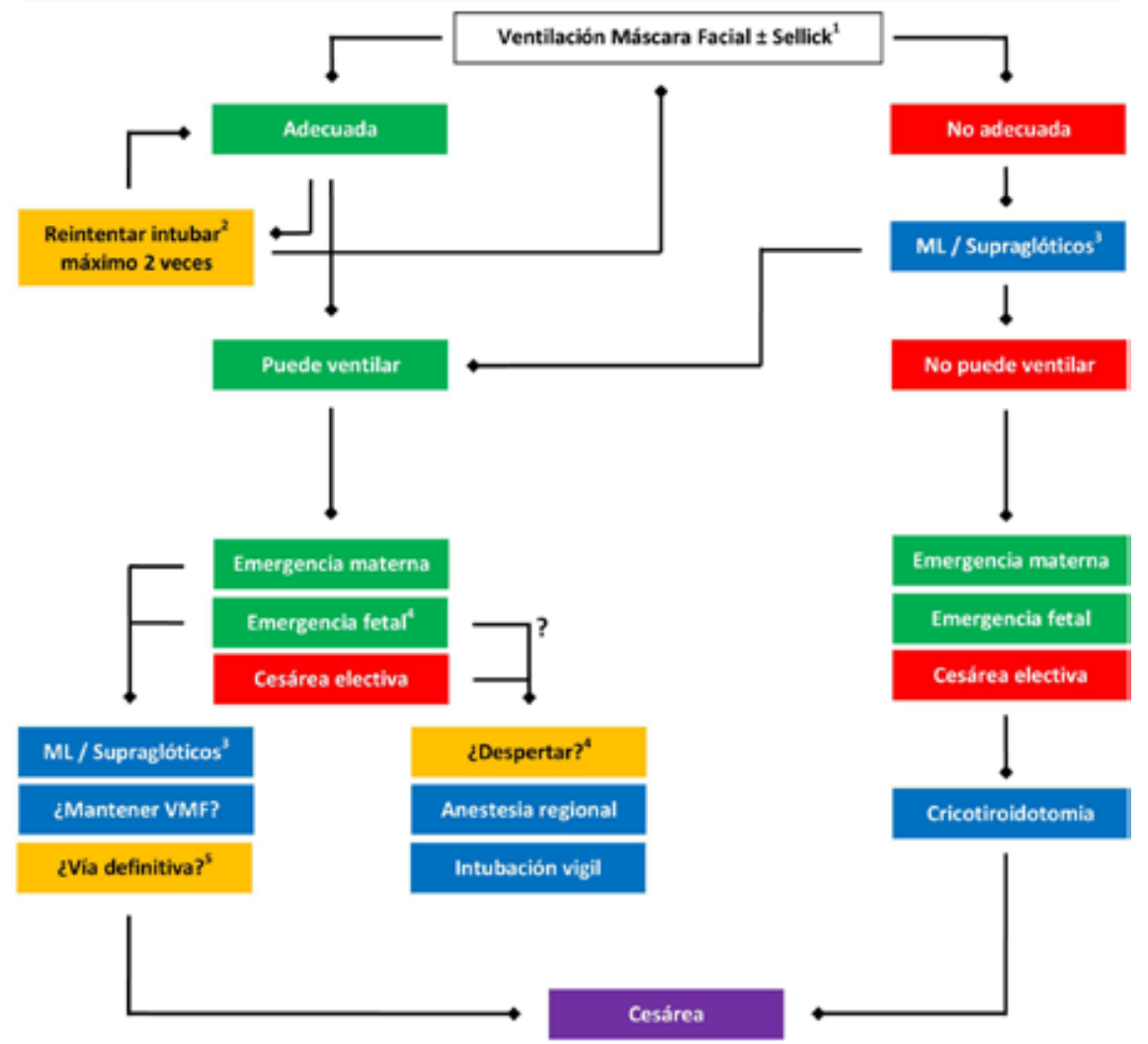

1 Presión cricoidea puede liberarse si afecta la visualización de la laringe o impide ventilación con máscara facial o laríngea. 2 Siempre y cuando no se haya traumatizado seriamente la vía aérea, se mantenga buena oxigenación y exista una alta probabilidad de éxito en otro intento, los cuales deberían realizarse usando otras técnicas como bougie, videolaringoscopia, etc. 3 Otros dispositivos supraglóticos considérese máscara laríngea Fast Track, Proseal, etc. 4 Seguridad materna es primero que fetal, debiendo evaluar cuidadosamente el riesgo de continuar el procedimiento con una vía aérea no protegida, con riesgo de desaturar y comprometer la vida de ambos, pudiendo en dicho caso despertar a la paciente e intentar un abordaje regional o intubación vigil aunque ponga en riesgo al feto. 5 Considerar de manera diferida en caso de que la emergencia materna no sea estabilizada inmediatamente post parto.

Figura 2. Algoritmo de manejo de vía aérea difícil en embarazada utilizado en Hospital Puerto Montt. Modificado de Balki et al (Anesthesiology 2012; 117(4):883-97) y Law et al (J Can Anesth 2013; 60:1089-118) con permiso de los autores. ML = Máscara Laríngea. VMF = Ventilación Máscara Facial. 


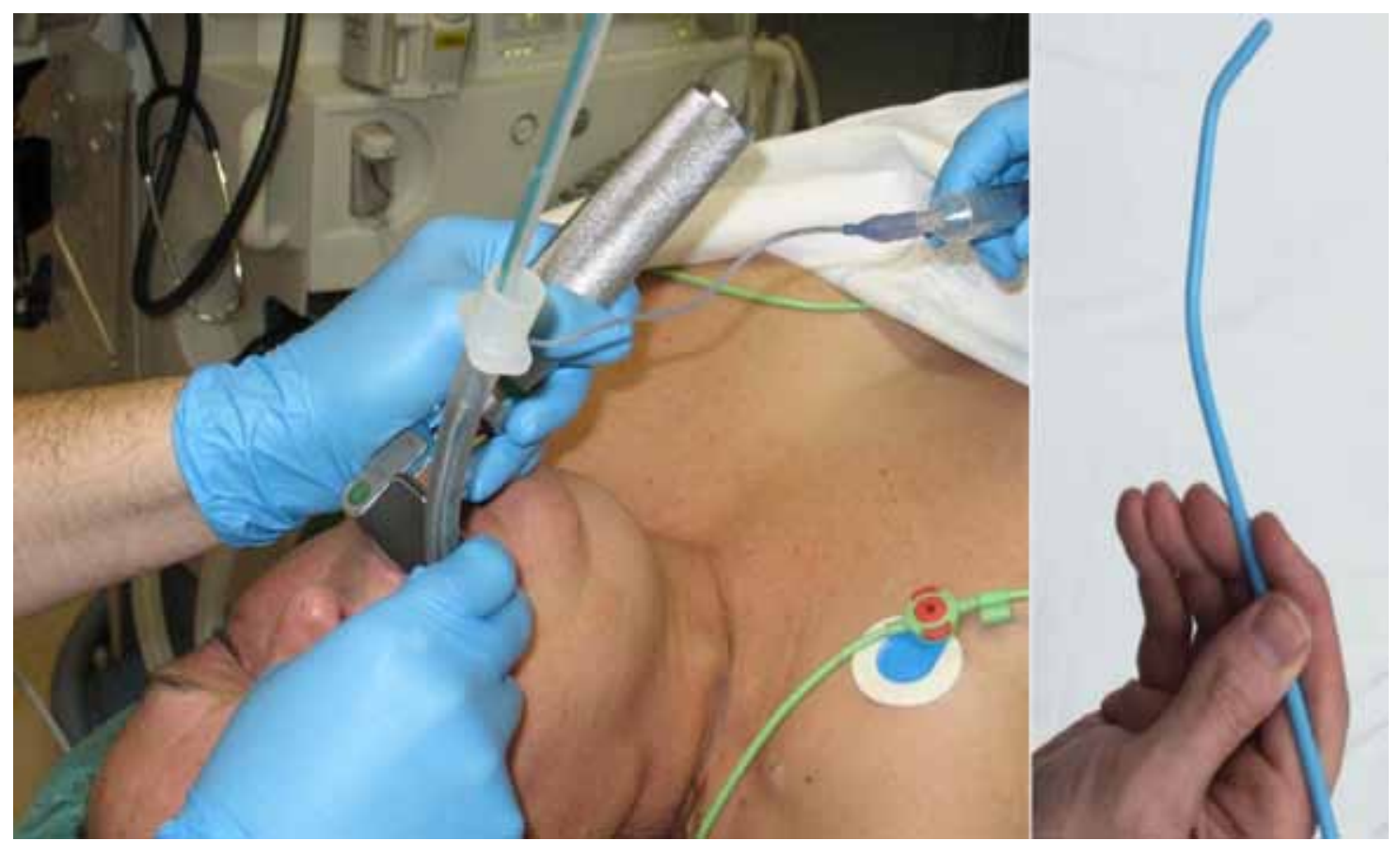

Figura 3. Ejemplo de intubación mediante uso de introductor de Eschmann o "gum elastic bougie".

Considerando lo previamente expuesto, y en pos del bienestar de la unidad materno-fetal, se ha replantado hoy en día el antiguo dogma que contraindicaba el apoyo ventilatorio inicial en pacientes con alto riesgo aspirativo. Múltiples estudios han mostrado una alta tasa de éxito para la mantención de la anestesia general en cesárea con apoyo ventilatorio inicial con máscara facial y/o laríngea, sin embargo, en nuestra realidad se reservan habitualmente para situaciones de emergencia (9).

En nuestro caso, y a pesar de los hallazgos clínicos descritos, afortunadamente se pudo asegurar la oxigenación de la unidad materno-fetal mediante laringoscopia directa con asistencia por Bougie (Figura 3).

Como abordajes alternativos recomendamos los dispositivos supraglóticos como la máscara laríngea cuya principal ventaja, a pesar de no proteger de aspiración, es que su curva de aprendizaje es relativamente corta inclusive en personal no médico.

En casos seleccionados, que no constituyan una urgencia, recomendamos realizar una intubación vigil con fibrobroncoscopia (14), videolaringoscopia (15) o inclusive establecer una vía aérea quirúrgica previa a la cesárea, todas las cuales han demostrado una tasa de éxito mayor en comparación a la intubación a ciegas $(12,13)$.

\section{CONCLUSIONES}

El síndrome de Hallermann-Streiff es una rara entidad y lo es aún más asociada a embarazo. La anestesia general para cesárea, sin ser la técnica de elección, es hoy en día una alternativa más segura para estas pacientes. En caso de sospechar una potencial vía aérea difícil, es mandatorio conocer el estado de la unidad materno-fetal para definir conductas (interrupción urgente o electiva), considerando una correcta selección de fármacos para la intubación así como la implementación de algoritmos específicos que incluyan el apoyo de nuevas tecnologías, todos las cuales, y en manos entrenadas, han permitido disminuir la morbi-mortalidad asociada a anestesia general y embarazo.

\section{REFERENCIAS}

1. Numabe $H$, Sawai $H$, Yamagata Z, Muto K, Kosaki R, Yuki $\mathrm{K}$, et al. Reproductive success in patients with Hallermann-Streiff syndrome. Am J Med Genet A 2011;155(9):2311-3.

2. Goldszmidt E. Principles and practices of obstetric airway management. Anesthesiol Clin 2008;26(1):10925.

3. de Souza DG, Doar LH, Mehta SH, Tiouririne M. Aspiration prophylaxis and rapid sequence induction for elective cesarean delivery: time to reassess old dogma?. Anesth Analg 2010;110(5):1503-5. 
4. Higurashi M, Oda M, lijima K, lijima S, Takeshita T, Watanabe N, et al. Livebirth: prevalence and follow-up of malformation syndrome in 27,472 newborns. Brain Dev 1990;12(6):770-3.

5. Hawkins JL, Chang J, Palmer SK, Gibbs CP, Callaghan WM. Anesthesia-related maternal mortality in the United States: 1979-2002. Obstet Gynecol 2011;117(1):69-74.

6. Bhatia N, Bhagat $H$, Sen I. Cricoid pressure: where do we stand? J Anaesthesiol Clin Pharmacol 2014;30(1):3-6.

7. Sharp LM, Levy DM. Rapid sequence induction in obstetrics revisited. Curr Opin Anaesthesiol 2009;22(3):357-61.

8. Tanoubi I, Drolet P, Donati F. Optimizing preoxygenation in adults. Can J Anesth 2009;56(6):449-66.

9. Murdoch $\mathrm{H}$, Scrutton $\mathrm{M}$, Laxton $\mathrm{CH}$. Choice of anaesthetic agents for caesarean section: a UK survey of current practice. Int J Obstet Anesth 2013;22(1):31-5.

10. Draisci G, Valente A, Suppa E, Frassanito L, Pinto R, Meo $\mathrm{F}$, et al. Remifentanil for cesarean section under general anesthesia: effects on maternal stress hormone secretion and neonatal well-being: a randomized trial. Int J Obstet Anesth 2008;17(2):130-6.
11. Balki M, Cooke ME, Dunington S, Salman A, Goldszmidt E. Unanticipated Difficult Airway in Obstetric Patients. Development of a new algorithm for formative assessment in high-fidelity simulation. Anesthesiology 2012;117(4):883-97.

12. Law JA, Broemling N, Cooper RM, Drolet $P$, Duggan LV, Griesdale DE, et al. Canadian Airway Focus Group. The difficult airway with recommendations for management--part 1--difficult tracheal intubation encountered in an unconscious/induced patient. Can J Anaesth 2013;60(11):1089-118.

13. Apfelbaum JL, Hagberg CA, Caplan RA, Blitt CD, Connis RT, Nickinovich DG, et al. Practice guidelines for management of the difficult airway: an updated report by the American Society of Anesthesiologists Task Force on Management of the Difficult Airway. Anesthesiology 2013;118(2):251-70.

14. Broomhead CJ, Davies W, Higgins D. Awake oral fibreoptic intubation for caesarean section. Int $\mathrm{J}$ Obstet Anesth 1995;4(3):172-4.

15. Kariya N, Kimura K, Iwasaki R, Ueki R, Tatara T, Tashiro $\mathrm{C}$. Intraoperative awake tracheal intubation using the Airway ScopeTM in caesarean section. Anaesth Intensive Care 2013;41(3):390-2. 